\title{
Distribución de aves acuáticas en las lagunas de oxidación de la ciudad de La Paz, Baja California Sur, México
}

\author{
Elvia Margarita Zamora-Orozco ${ }^{1}$, Roberto Carmona ${ }^{1,2}$ \& Georgina Brabata ${ }^{1}$ \\ 1 Universidad Autónoma de Baja California Sur, Departamento de Biología Marina, Apdo. Postal 19-B, La Paz, Baja \\ California Sur, C.P. 23080, México. Fax (612) 1-28-08-01; beauty@uabcs.mx \\ 2 Universidad Autónoma de Baja California, Facultad de Ciencias Marinas, Apdo. Postal 1653, Ensenada, Baja \\ California
}

Recibido 20-III-2002. Corregido 13-IX-2006. Aceptado 12-I-2007.

\begin{abstract}
Distribution of aquatic birds in oxidation lagoons of La Paz city in South Baja California, Mexico. Taxonomic composition, spatial and temporal distribution of aquatic birds in oxidation lagoons (LO) of La Paz city in south Baja California, Mexico, were determined during 24 censuses realized in two-week intervals (April/98-March/99). There are five lagoons of $5 \mathrm{Ha}$ each and 17 ha of terrains constantly flooded that serve as feeding areas for cattle and birds. One hundred twenty three species were observed, 75 of which were aquatic birds. A total of 46041 observations were made (average 1918 birds/census). Richness and abundance of aquatic birds were influenced mainly by migration of anatids and sandpipers. The first group had the greatest abundance due to its affinity towards fresh water bodies. The terrains were the favorite sites of dabbling ducks (Anas) and sandpipers. In contrast, two of the most abundant species (Oxyura jamaicensis, $12.5 \%$ of all species, and Fulica americana, $8.8 \%$ ) restricted their presence to the oxidation lagoons. LO presented a bird structure of its own and atypical, according to the dryness of the region. Rev. Biol. Trop. 55 (2): 617-626. Epub 2007 June, 29.
\end{abstract}

Key words: Aquatic birds, migratory birds, distribution, Baja California, oasis.

Las migraciones que la mayoría las aves neárticas llevan a cabo les permiten pasar el invierno en zonas menos extremosas (Kasprzyk y Harrington 1989). Para realizar estos movimientos, utilizan una serie de sitios eslabonados, por lo que la desaparición de alguno de ellos, puede ocasionar un notorio efecto negativo acumulado (Myers et al. 1987). Una parte importante de las áreas de paso e invernación se encuentran en los trópicos y subtrópicos, donde en general, existen pocos estudios (Rubio et al. 1997).

La Península de Baja California, se ubica en la porción noroccidental de la República Mexicana y forma parte del Corredor Migratorio del Pacífico (Carmona y Danemann 1998). La región sur de dicha península, se caracteriza por un clima seco subtropical, con precipitación media anual menor de $200 \mathrm{~mm}$ (García y
Mosiño 1969). Dada la aridez de la región, los ambientes dulceacuícolas son escasos (Guzmán et al. 1994). Al sur de la península se localiza la región de La Paz, en ella no se cuenta con cuerpos dulceacuícolas naturales; sin embargo, se tienen registros de la avifauna en dos zonas artificiales, el embalse de aguas tratadas del ejido El Centenario y las lagunas de oxidación de la Ciudad de La Paz (LO) (Carmona et al. 1999, Castillo-Guerrero y Carmona 2001).

En las últimas décadas, el constante incremento demográfico en el extremo sur de la península, ha provocado alteraciones de hábitat en diferentes localidades, incluida la Ensenada de La Paz (Guzmán et al. 1994). Los desechos que producen los asentamientos urbanos, en especial las aguas residuales, pueden resultar perjudiciales para la naturaleza. Por lo que, la limpieza de estas aguas es un objetivo constante 
del gobierno. En la ensenada de La Paz, hasta 1985, la limpieza se efectuaba a través de cinco lagunas de oxidación (en adelante L1 a L5) de 5 ha cada una; actualmente no son utilizadas, pero permanecen con agua (Carmona et al. 1999). Las lagunas han conformado un ecosistema dulceacuícola, utilizado por un número importante de aves migratorias y residentes (Zamora-Orozco 2001). Dada la capacidad de desplazamiento de la mayoría de las aves acuáticas, los ambientes dulceacuícolas aledaños deben ser considerados como parte del mosaico paisajístico que la región ofrece a estos organismos (Castillo-Guerrero y Carmona 2001).

A pesar de la aparente importancia que LO representa para las aves, ningún estudio sistemático ha sido publicado a este respecto. El trabajo presente se abocó a determinar la distribución temporal y espacial de las aves acuáticas en la zona, durante un ciclo anual.

\section{MATERIALES Y MÉTODOS}

La Bahía de La Paz, se localiza en la costa sudoriental de la Península de Baja California, en el Noroeste de México. La bahía presenta un clima semidesértico, con una temperatura media anual de $23{ }^{\circ} \mathrm{C}$ y una precipitación media anual de menos de $200 \mathrm{~mm}$. Los vientos del sur dominan de abril a agosto, mientras que a partir de octubre y hasta marzo, dominan los vientos del noroeste (Jiménez 1983).

Las cinco lagunas de oxidación se localizan en las afueras de la ciudad de La Paz, por la carretera al norte $\left(24^{\circ} 6^{\prime} 35.7^{\prime \prime} \mathrm{N}\right.$ y $110^{\circ} 20^{\prime} 59.1$ ' W), suman 25 ha de espejo de agua, además su superficie se incrementa por 17 ha de terrenos aledaños (TA), que son constantemente inundados. Estas lagunas presentan una profundidad que va de $\operatorname{los} 3 \mathrm{~cm}$ a $2.5 \mathrm{~m}$, además entre ellas hay un gradiente de profundidad donde $\mathbf{L} \mathbf{1}$ es la más somera y L5) la más profunda. Alrededor de ellas crece vegetación de desierto (como mezquite, palo verde y frutilla, entre otros) y árboles exóticos (eucalipto). Durante cinco meses del estudio (noviembre a marzo), L4 y $\mathbf{L 5}$ permanecieron secas, por cuestiones de limpieza por parte del personal de la planta de tratamiento.

En las cinco lagunas y los terrenos aledaños se efectuaron censos con periodicidad quincenal, durante un ciclo anual (abril de 1998 a marzo de 1999). En cada ocasión se contó e identificó a las aves presentes. Los recorridos se realizaron siempre al amanecer, paralelos al margen mayor de cada una de las lagunas; desde $\mathbf{L 1}$ hasta L5. Los conteos se realizaron con binoculares $(8 \mathrm{x}$ y $10 \mathrm{x})$ y telescopios (1560x), a una distancia no mayor de $100 \mathrm{~m}$ de los grupos de aves. Las aves se contaron directamente cuando sus números lo permitieron y en el caso de bandadas mayores a 300 individuos, la abundancia se estimó con base en el método de Page et al. (1979), con las sugerencias de Kasprzyk y Harrington (1989), respecto a la estandarización de las estimaciones.

Se estimaron las abundancias promedio mensuales, mismas que fueron divididas por grupo funcional. Respecto a la riqueza (también por grupo funcional), ésta se presenta como el total de especies vistas en cada mes. Adicionalmente, se calculó el promedio mensual de las cuatro especies numéricamente más importantes (más del $9 \%$ de la abundancia total).

\section{RESULTADOS}

Se observaron 75 especies de aves acuáticas (Cuadro 1) y 48 terrestres (Apéndice I), pertenecientes a 13 órdenes, 34 familias y 82 géneros. Respecto a las acuáticas, 26 especies fueron residentes y 49 migratorias (Cuadro 1).

Durante el año se acumuló un total de 46 041 registros de estas aves. Los grupos más abundantes fueron anátidos y aves playeras con 44.1 y $31.7 \%$ del total acumulado, respectivamente. Las familias de aves acuáticas con mayor número de especies fueron: Ardeidae (11), Anatidae (19), Charadriidae (5), Scolopacidae (14) y Laridae (10) (Cuadro 1). Por otro lado, siete de estas especies están dentro de alguna de las categorías de protección definidas por el Gobierno Mexicano (Semarnat 2002), una en peligro de extinción y seis requieren protección 
CUADRO 1

Especies de aves acuáticas observadas en la zona de estudio

TABLE 1

Species of aquatic birds observed in the study area

Especie

Tachybaptus dominicus*

Podilymbus podiceps*

Podiceps nigricollis

Pelecanus erythrorhynchos

Pelecanus occidentalis

Fregata magnificens

Ardea herodias

Ardea alba

Egretta thula

Egretta caerulea

Egretta tricolor

Egretta rufescens

Bubulcus ibis

Butorides virescens

Nycticorax nycticorax

Nyctanassa violacea*

Eudocimus albus

Plegadis chihi

Dendrocygna autumnalis

Anser albifrons

Chen caerulescens

Cairina moschata

Anas crecca

Anas platyrhynchos

Anas acuta

Anas discors

Anas cyanoptera

Anas clypeata

Anas strepera

Anas penelope

Anas americana

Aythya valisineria

Aythya americana

Aythya collaris

Aythya affinis

Melanitta perspicillata

Oxyura jamaicensis

Pandion haliaetus

Fulica americana*

Pluvialis squatarola
Frecuencia de observación Registros total

N.O.M. Condición de la especie

24

120

14

487

150

24

142

35

51

148

2

16

3

2039

17

9

25

1

2869

90

14

4

3

461

115

2940

985

4365

3365

124

4

249

4

711

49

827

4

6022

2

4246

21
Pr

$\mathrm{R}$

RM

MI

MI

R

P

Pr R

RM

RM

RM

RM

RM

R

R

R

R

$\mathrm{R}$

MV

MI

MT

MT

MT

MI

MT

MI

MI

MI

MI

MI

MT

MT

MT

MI

MT

MI

MI

MV

R

RM

MT 
CUADRO 1 (Continuación)

Especies de aves acuáticas observadas en la zona de estudio

TABLE 1 (Continued)

Species of aquatic birds observed in the study area

Especie

Charadrius alexandrinus

Charadrius wilsonia

Charadrius semipalmatus

Charadrius vociferus*

Haematopus palliatus

Himantopus mexicanus*

Recurvirostra americana*

Tringa melanoleuca

Tringa flavipes

Catoptrophorus semipalmatus

Actitis macularia

Numenius phaeopus

Numenius americanus

Limosa fedoa

Calidris canutus

Calidris alba

Calidris mauri

Calidris minutilla

Calidris alpina

Limnodromus spp.

Phalaropus tricolor

Larus atricilla

Larus pipixcan

Larus philadelphia

Larus delawarensis

Larus californicus

Larus livens

Larus occidentalis

Sterna caspia

Sterna maxima

Sterna elegans

Ceryle alcyon

Sayornis nigricans

Seiurus noveboracensis

Geothlypis beldingi

Frecuencia de observación Registros total

N.O.M.

Condición de la especie

12

35

268

369

13

2034

1322

310

288

255

75

30

13

824

3

11

4271

999

110

9

478

28

3

26

3

16

3

2

1

25

1

2
RM

$\mathrm{R}$

MI

$\mathrm{R}$

$\mathrm{R}$

RM

RM

MI

MI

MV

MI

MV

MV

MV

MI

MI

MI

MI

MI

MI

MT

MT

MT

MI

MI

MI

R

Pr

MI

MI

MI

MI

MT

R

MI

R

Frecuencia de observación (máximo 24), total de registros, categoría en la Norma Oficial Mexicana 2001 (P, en peligro de extinción; A, amenazada; $\mathbf{P r}$, con protección especial) y condición temporal en el área ( $\mathbf{R}$, residente con población reproductora; $\mathbf{P}$, permanentemente observable durante la mayor parte del año, pero sin poblaciones reproductoras; $\mathbf{M I}$, migratorio invernante observable sólo una parte del año incluido el invierno; MT, migratorio transeúnte, observable sólo una parte del año sin permanencia invernal; RM, población residente incrementada con individuos migratorios; $\mathbf{M V}$, migratorio con fracciones poblacionales presentes todo el año, sin reproducción local). El asterisco posterior al nombre científico indica su registro reproductivo en las lagunas. 
especial (Cuadro 1). Adicionalmente dentro de las aves terrestres observadas dos se consideran amenazadas y tres bajo protección especial (Semarnat 2002; Apéndice 1).

La abundancia mostró fluctuaciones, con los menores números en primavera-verano (abril a julio), en otoño temprano inició un ascenso, con una relativa estabilización entre octubre y febrero, a partir de marzo los números disminuyeron (Fig. 1A). Al dividir la abundancia por grupo funcional, se observó que los playeros fueron los primeros en arribar al área, en julio, con sus mayores abundancias entre agosto y noviembre, posteriormente sus números declinaron, para prácticamente aban- donar la zona en febrero (Fig. 1A). Por su parte, los anátidos iniciaron su llegada entre septiembre y octubre, con sus números más altos en otoño (agosto a noviembre), a lo largo del invierno sus abundancia disminuyeron, con un notorio decremento a inicios de primavera (marzo; Fig. 1A). Por su parte, los ardéidos, siempre en números modestos, alcanzaron su máxima abundancia entre octubre y diciembre, y sus mínimos en el verano (Fig 1A). Los rálidos, que estuvieron representados por una sola especie (Fulica americana Gmelin, 1789), alcanzaron sus abundancias mayores entre mediados de otoño e invierno (octubre a febrero; Fig. 1A). Los láridos, aunque en bajos
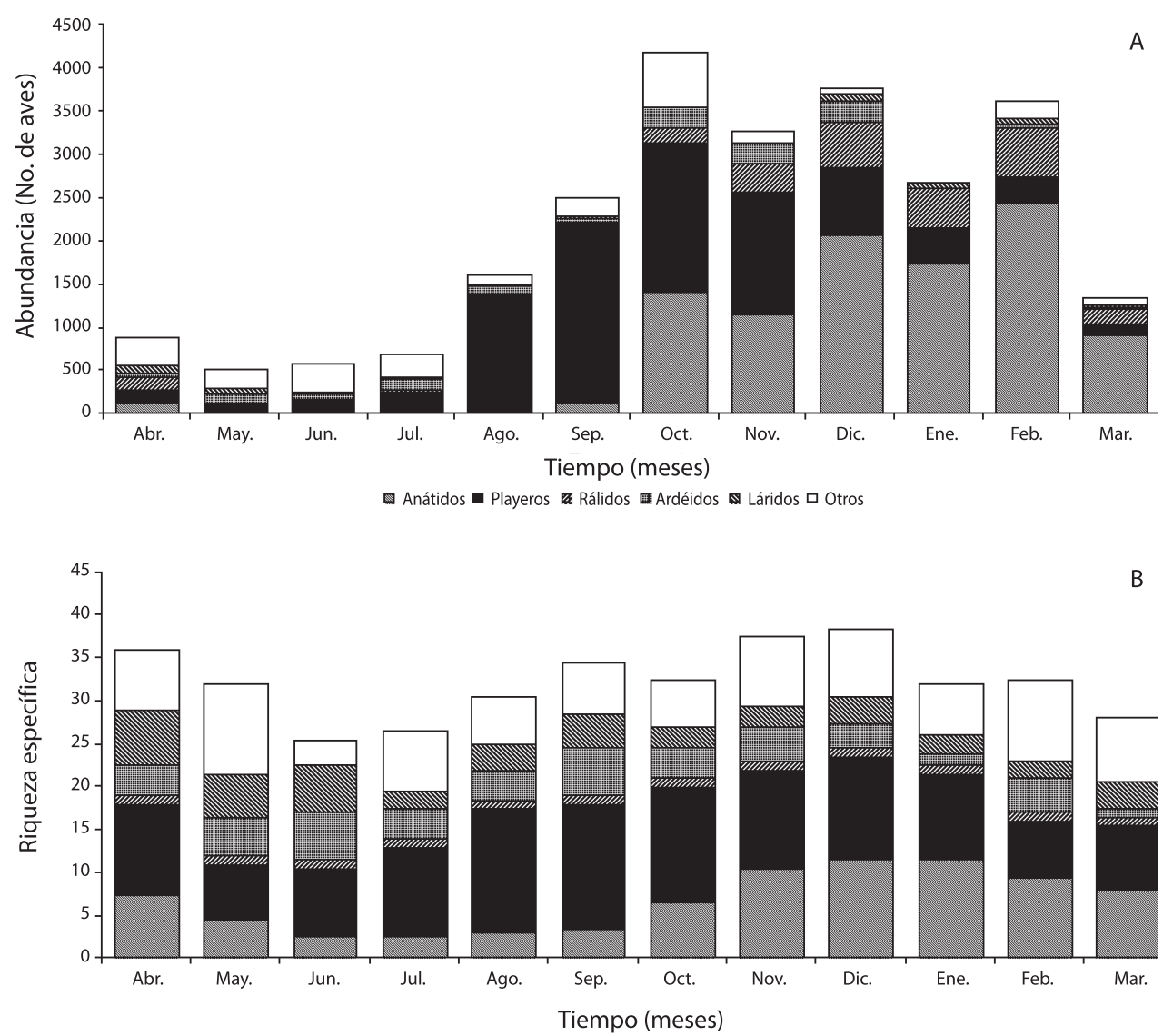

Fig. 1. A. Abundancia mensual promedio de aves acuáticas por grupo funcional observadas en las Lagunas de Oxidación. B. Riqueza específica mensual de aves acuáticas por grupo funcional observadas en las Lagunas de Oxidación.

Fig. 1. A. Monthly abundance average of aquatic birds by functional group, in the Oxidation Ponds. B. Monthly specific richness of aquatic birds by functional group observed in the Oxidation Ponds. 
números, se presentaron a lo largo de todo el año (Fig. 1A).

La riqueza mostró sus valores menores en el verano (junio y julo), con un incremento en agosto, entre septiembre y diciembre las riquezas se mantuvieron altas y relativamente estables, entre enero y marzo tendieron a disminuir, con repuntes en primavera (abril y mayo; Fig. 1B).

Por grupo funcional, la riqueza de los anátidos presentó sus valores máximos entre noviembre y febrero y la menor durante el verano y el otoño temprano (mayo a septiembre). Los playeros estuvieron presentes durante todo el año, con sus valores máximos entre el otoño temprano y mediados del invierno (agosto-diciembre), a partir del cual disminuyeron, para alcanzar sus menores riquezas en verano (mayo-junio). Los grupos restantes mantuvieron un número de especies relativamente homogéneo a lo largo del año (Fig. 1B).

Las cuatro especies numéricamente más importantes, fueron el pato rojo (Oxyura jamaicensis Gmelin, 1789) con $13 \%$ de la abundancia total, el pato canelo (Anas cyanopteraVieillot, 1816) con $9.5 \%$, el playerito occidental (Calidris mauri Cabanis, 1857, 9.3 $\%)$ y la gallareta gris (F. americana, $9.2 \%$ ). En general se observaron dos tendencias, en la primera se incluyen el pato rojo, el pato canelo y la gallareta gris; estas aves aparecieron en el otoño temprano (septiembre-octubre), con sus máximos números entre el otoño tardío (noviembre) y el invierno (febrero), a inicios de la primavera (marzo) las tres especies disminuyeron sus abundancias (Fig. 2A, B, D). La segunda tendencia sólo incluyó al playerito occidental, especie que únicamente estuvo bien representada en el otoño temprano (agosto y septiembre) (Fig. 2C).

\section{DISCUSIÓN}

En LO la riqueza global de la avifauna acuática fue alta (75 especies); al compararla con la registrada para otras zonas peninsulares, como el estero de San José con 50 especies
(Guzmán et al. 1994), ríos Mayor y Hardy con 62 especies (Ruiz-Campos y Rodríguez-Meraz 1997) y la salina de Guerrero Negro con 79 especies (Carmona y Danemann 1998). Lo que se resalta aún más al considerar que el área de estudio alberga esta riqueza en una superficie mucho menor (ca. 45 ha), a la que presentan cualquiera de las zonas antes mencionadas.

De la misma forma que en la zona costera de la Ensenada de La Paz y que prácticamente la totalidad de los humedales de la península, la mayoría de las especies observadas fueron migratorias (vgr. Guzmán et al. 1994, Carmona y Danemann 1998, Castillo-Guerrero y Carmona 2001). Así, LO es utilizada como sitio de escala y recuperación y como área de invernación por las aves migratorias. Además, es una zona de alimentación y refugio de especies locales durante todo el año, gracias al riego constante que recibe la comunidad vegetal adyacente. Sin embargo, en la mayor parte de los humedales peninsulares, el grupo numéricamente más importante es el de las aves playeras (Page et al. 1997, Carmona et al. 2004), en contraste en LO el grupo dominante fue el de los anátidos, lo que se relaciona con la presencia de agua dulce.

El incremento poblacional humano conlleva una reducción de hábitats óptimos para las aves nativas, las cuales son desplazadas e incluso erradicadas (Myers et al. 1987, Llinas y Jiménez 1997). Pero, como en el caso de LO, es posible compensar esta pérdida mediante la implementación de áreas artificiales (Myers et al. 1987, Schnack et al. 2000), demostrando que algunas actividades humanas son compatibles con la conservación; una situación semejante se encuentra en el embalse de El Centenario, área que también alberga una comunidad aviar diversa (Castillo-Guerrero y Carmona 2001). El alto número de especies observadas se relaciona con la coexistencia en la zona de aves marinas, dulceacuícolas y terrestres, lo que en una región típicamente árida como la península, contrasta con el resto de los ambientes.

En lo que se refiere a las especies más abundantes: 

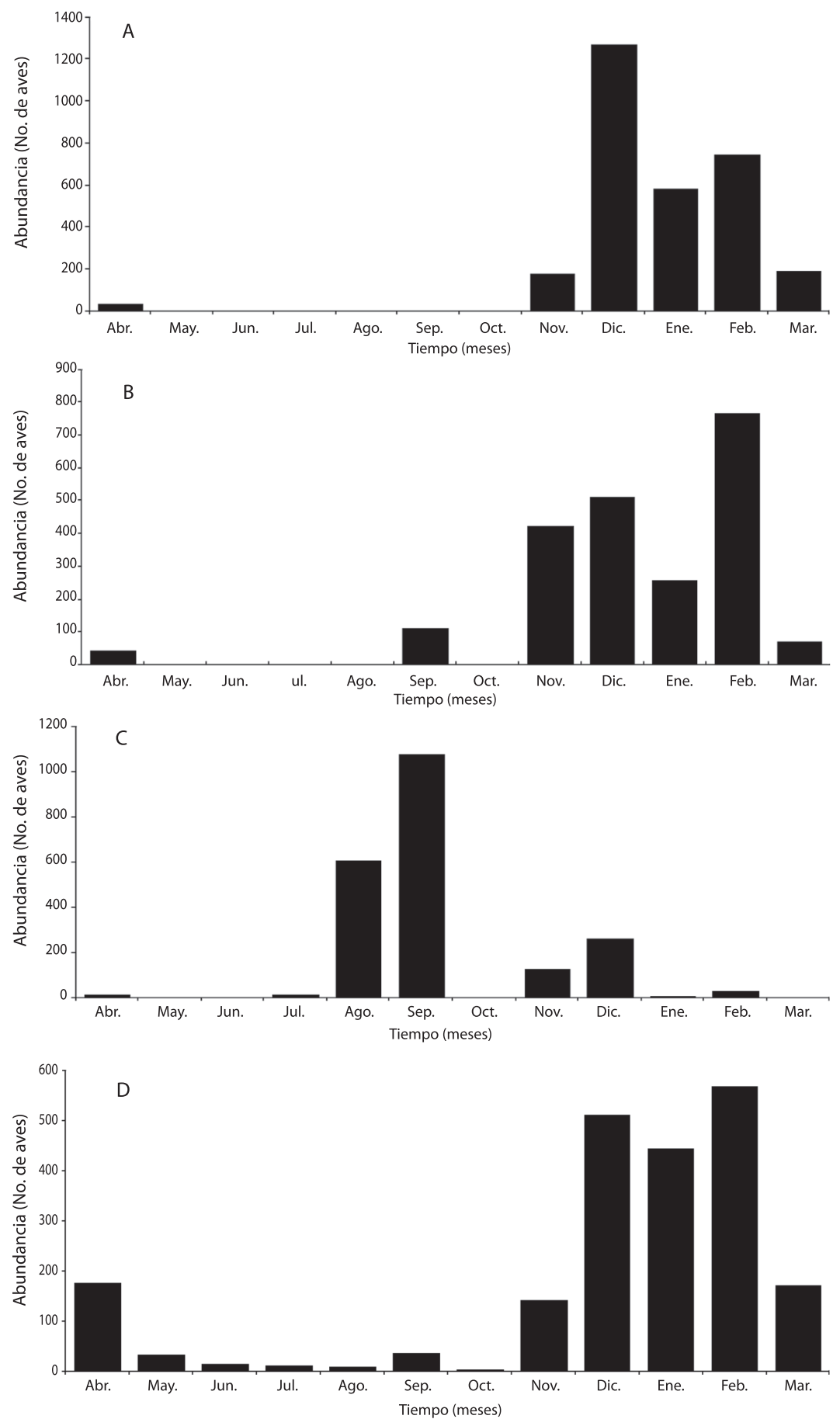

Fig. 2. Abundancia mensual promedio de las cuatro especies más numerosas: A. Pato rojo (O. jamaicensis), B. Pato canelo (A. cyanoptera), C. Playerito occidental (C. mauri), D. Gallareta gris (F. americana); en las Lagunas de Oxidación.

Fig. 2. Monthly abundance average of the four species more abundant: A. Ruddy Duck (O. jamaicensis), B. Cinnamon Teal (A. cyanoptera), C. Western Sandpiper (C. mauri), D. American Coot (F. americana); in the Oxidation Ponds. 
Pato rojo (O. jamaicensis): la presencia de estos organismos en LO tuvo una tendencia semejante a la registrada por Guzmán et al. (1994), en el estero de San José del Cabo y recientemente, por Castillo-Guerrero y Carmona (2001) en el embalse de El Centenario, ya que en todos los casos se registraron las mayores abundancias en enero. En LO sus números variaron de 1800 individuos en enero a 50 en la segunda quincena de mayo; como población invernante sumaron aproximadamente 600 individuos, cabe señalar que los números observados en el trabajo presente son los más altos registrados para la península.

Pato canelo (A. cyanoptera): el comportamiento en las abundancias de esta especie indica que la zona fue básicamente utilizada como sitio de paso y recuperación durante sus movimientos migratorios (otoño y primavera), patrón semejante al observado por Castillo Guerrero (2000).

Playerito occidental (C. mauri): LO es utilizada aparentemente como una zona alterna de alimentación por individuos que van de paso por la Ensenada de La Paz (Carmona et al. 2003). Durante el otoño, dados sus mayores requerimientos energéticos causados por la migración y la muda de plumas primarias en los adultos (O'Reilly y Wingfield 2003), la presencia de lugares alternos de alimentación no influenciados por el nivel de marea, como LO, cobra particular importancia.

Gallareta gris (F. americana): En LO se presentó todo el año, lo que coincidió con lo visto en el Estero San José del Cabo (Guzmán et al. 1994) y en el embalse de El Centenario (Castillo-Guerrero y Carmona 2001). En el estero, sus mayores abundancias fueron en el verano, mientras que en $\mathbf{L O}$ y el embalse, ocurrieron en el periodo otoño-invierno, lo cual indica que la primera zona es importante para su reproducción y las dos últimas para su paso e invernacón (Castillo Guerrero y Carmona 2001).

En suma, LO fue utilizada fundamentalmente como una zona de paso otoñal por las aves playeras y como un área de invernación por los anátidos y los rálidos.

\section{AGRADECIMIENTOS}

Se agradecen las facilidades otorgadas por la Comisión Nacional del Agua por el acceso irrestricto a sus instalaciones. También se agradece a Gorgonio Ruiz, Jorge Llinas y José Alfredo Castillo pos sus atinadas sugerencias y a todas las personas que ayudaron en el campo.

\section{RESUMEN}

Se determinó la composición taxonómica y la distribución espacial y temporal de aves acuáticas de las lagunas de oxidación (LO), de la ciudad de La Paz, Baja California Sur, México, durante 24 censos quincenales (abril/98marzo/99). Se trata de cinco lagunas, de cinco ha cada una y 17 ha de terrenos aledaños constantemente inundados que sirven como zona de alimentación para el ganado y las aves. Se observaron 123 especies de aves, de las cuales 75 fueron acuáticas. En total se realizaron 46041 registros (promedio= 1918 aves/censo). La riqueza y la abundancia estuvieron influenciadas por la migración, principalmente de anátidos y playeros, los primeros fueron el grupo más abundante, debido a su afinidad por cuerpos dulceacuícolas. Los terrenos aledaños fueron los preferidos por los patos vadeadores (Anas) y por los playeros. En contraste, dos de las especies más abundantes (Oxyura jamaicensis $12.5 \%$ del total y Fulica americana $8.8 \%$ ), restringieron su presencia al espejo de agua. LO presentó un componente aviar propio y atípico, dada la aridez de la región.

Palabras clave: Aves acuáticas, aves migratorias, distribución, Baja California, oasis.

\section{REFERENCIAS}

Carmona, R. \& G. Danemann. 1998. Distribución espaciotemporal de aves en la salina de Guerrero Negro, Baja California Sur, México. Cien. Mar. 24: 389-408.

Carmona, R., J.A. Castillo-Guerrero \& E.M. ZamoraOrozco. 1999. Registros recientes del Zambullidor menor (Tachybaptus dominicus) y diez especies de anátidos en las adyacencias de la Bahía de La Paz, Baja California Sur, México. An. Inst. Biol. Univ. Nal. Autón. México. serie Zool. 70: 191-203.

Carmona, R., A. Álvarez, A. Cuéllar-Brito \& M. ZamoraOrozco. 2003. Uso estacional de dos áreas, marina y dulceacuícola, por aves playeras en función al nivel de marea, en Baja California Sur, México. Ornitol. Neotrop. 14: 201-214. 
Castillo-Guerrero, J.A. 2000. Composición temporal de aves acuáticas en el tanque de almacenamiento de aguas tratadas, ejido El Centenario, Municipio de La Paz, B.C.S., México. Tesis de Licenciatura, Universidad Autónoma de Baja California Sur, La Paz, Baja California Sur, México. 53 p.

Castillo-Guerrero, J.A. \& R. Carmona. 2001. Distribución espacio-temporal de aves acuáticas y rapaces en un embalse dulceacuícola artificial, en las adyacencias de la bahía de La Paz, Baja California Sur, México. Biol. Trop. 49: 1055-1066.

Castillo-Guerrero, J.A., E.M. Zamora Orozco \& R. Carmona. 2002. Aves acuáticas anidantes en dos cuerpos dulceacuícolas artificiales, adyacentes a la Ciudad de La Paz, Baja California Sur, México. Hidrobiológica 12: 85-87.

Fernández, G., R. Carmona \& H. de la Cueva. 1998. Abundance and seasonal variation of Western sandpipers (Calidris mauri) in Baja California Sur, México. Southwest. Nat. 43: 57-61.

García, E. \& P. Mosiño. 1969. Los climas de Baja California. Universidad Nacional Autónoma de México, México DF, México. p. 29-55.

Grinnell, J. 1928. A distributional summation of the ornithology of Lower California. U. Calif. Publ. Zool. 32: $1-300$.

Guzmán, J., R. Carmona, E. Palacios \& M. Bojórquez. 1994. Distribución temporal de aves acuáticas en el estero de San José del Cabo, B.C.S., México. Cien. Mar. 20: 93-103.

Jiménez, A.R. 1983. Aplicación de un modelo hidrodinámico numérico a la Ensenada de La Paz, B.C.S. Tesis de Maestría, Centro de Investigación Científica y Educación Superior de Ensenada, Ensenada, Baja California Sur, México. 78 p.

Kasprzyk, M.J. \& B.A. Harrington. 1989. Manual de campo para el estudio de playeros. Red Hemisférica de reservas para aves playeras, Ensenada, Baja California Sur, México. 134 p.

Llinas, J. \& M.L. Jiménez. 1997. Recent records of the least grebe Tachybaptus dominicus in Baja California Sur, México. An. Inst. Biol. UNAM Ser. Zool. 68: 187-191.

Myers, J.P., R.I.G. Morrison, P.Z. Antas, B.A. Harrington, T.E. Lovejoy, M. Sallaberry, S.E. Senner \& A. Tarak.
1987. Conservation strategy for migratory species. Am. Sci. 75: 19-26.

Nagarajan, R. \& K. Thiyagesan. 1996. Waterbirds and substrate quality of the Pichavaram wetlands, southern India. Ibis 138: 710-721.

O’Reilly, K.M. \& J.C. Wingfield. 2003. Seasonal, age, and sex differences in weight, fat reserves, and plasma corticosterone in Western Sandpipers. Condor 105: 13-26.

Page, G.W., L.E. Stenzel \& C.M. Wolfe. 1979. Aspects of the occurrence of shorebirds on a Central California Estuary, p. 15-22. In F.A. Pitelka (ed.). Studies in avian biology. Cooper Ornithological Society, Allen, Kansas, Kansas, EEUU.

Rubio, L., R. Rodriguez-Estrella \& E. Pineda. 1997. El uso del hábitat por aves residentes e invernantes. p. 221248. In L. Arriaga \& R. Rodríguez-Estrella (eds.). Los Oasis de la Península de Baja California. SIMACCIBNOR, La Paz, Baja California Sur, México.

Ruiz-Campos, G. \& M. Rodríguez-Meraz. 1997. Composición taxonómica y ecológica de la avifauna de los ríos El Mayor y Hardy y áreas adyacentes en el valle de Mexicali, Baja California, México. An. Inst. Biol. UNAM Ser. Zool. 68:291-315.

Schnack, J.A., F.O. De Francesco, U.R. Colado, M.L. Novoa \& E.J. Schnack. 2000. Humedales antrópicos: su contribución para la conservación de la biodiversidad en los dominios subtropical y pampásico de la Argentina. Ecol. Austral 10: 63-80.

Secretaría de Medio Ambiente y Recursos Naturales (Semarnat). 2002. Norma Oficial Mexicana NOM059-ECOL-2001. Diario Oficial de la Federación, Ciudad de México, México, 6 de marzo de 2002, Tomo DLXXXII: 1-56.

White, D.H. \& D. James. 1978. Differential use of fresh water environments by wintering waterfowl of coastal Texas. Wilson Bull. 90: 99-111.

Wilbur, S.R. 1987. Birds of Baja California. University of California, Berkeley, California, EEUU. 253 p.

Zamora-Orozco, E.M. 2001. Distribución espacio-temporal de aves acuáticas en las lagunas de oxidación de la ciudad de La Paz, B.C.S., México. Tesis de Licenciatura, Universidad Autónoma de Baja California Sur, La Paz, EEUU. 60 p.

Zar, J.H. 1999. Biostatistical analysis. Prentice Hall, Nueva York, EEUU. 718 p. 


\section{APÉNDICE I}

Aves terrestres observadas durante el estudio

\section{APPENDIX 1}

Land birds observed during the study

\begin{tabular}{|c|c|c|c|c|c|}
\hline Especies & N.O.M & Estatus & Especies & N.O.M & Estatus \\
\hline Cathartes aura & & $\mathrm{R}$ & Campilorhynchus brunneicapillus & & $\mathrm{R}$ \\
\hline Accipiter gentilis & $\mathrm{A}$ & M & Mimus polyglottos & & $\mathrm{R}$ \\
\hline Buteo swansoni & $\operatorname{Pr}$ & M & Toxostoma cinereum & & $\mathrm{R}$ \\
\hline Buteo jamaicensis & & $\mathrm{R}$ & Anthus rubescens & & M \\
\hline Caracara cheriway & & $\mathrm{R}$ & Phainopepla nitens & & $\mathrm{R}$ \\
\hline Falco sparverius & & $\mathrm{R}$ & Sturnus vulgaris & & M \\
\hline Falco peregrinus & $\operatorname{Pr}$ & M & Vermivora celata & & M \\
\hline Falco mexicanus & $\mathrm{A}$ & M & Dendroica petechia & & M \\
\hline Zenaida asiatica & & $\mathrm{R}$ & Dendroica coronata & & M \\
\hline Zenaida macroura & & $\mathrm{R}$ & Cardinalis cardinalis & & $\mathrm{R}$ \\
\hline Columbina passerina & & $\mathrm{R}$ & Cardinalis sinuatus & & $\mathrm{R}$ \\
\hline Geococcyx californianus & & $\mathrm{R}$ & Pheucticus melanocephalus & & M \\
\hline Calypte costae* & & $\mathrm{R}$ & Chondestes grammacus & & M \\
\hline Melanerpes uropygialis & & $\mathrm{R}$ & Amphispiza bilineata & & $\mathrm{R}$ \\
\hline Picoides scalaris & & $\mathrm{R}$ & Passerculus sandwichensis rostratus & $\operatorname{Pr}$ & $\mathrm{R}$ \\
\hline Colaptes chrysoides & & $\mathrm{R}$ & Zonotrichia leucophris & & M \\
\hline Contopus sordidulus & & M & Agelaius phoeniceus & & M \\
\hline Pirocephalus rubinus & & $\mathrm{R}$ & Xantocephalus xantocephalus & & M \\
\hline Myarchus cinerascens & & $\mathrm{R}$ & Euphagus cyanocephalus & & M \\
\hline Progne subis & & M & Molotrus ather & & $\mathrm{R}$ \\
\hline Tachycineta bicolor & & M & Icterus cucullatus & & $\mathrm{R}$ \\
\hline Tachycineta thalassina & & $\mathrm{R}$ & Icterus parisorum & & $\mathrm{R}$ \\
\hline Corvus corax & & $\mathrm{R}$ & Carpodacus mexicanus & & $\mathrm{R}$ \\
\hline Auriparus flaviceps & & $\mathrm{R}$ & Carduelis psaltria & & $\mathrm{R}$ \\
\hline
\end{tabular}

Se incluye su categoría en la Norma Oficial Mexicana 2001 ( $\mathbf{P}=$ en peligro de extinción, $\mathbf{A}=$ amenazada y $\mathbf{P r}=$ protección especial) y su estatus en la zona ( $\mathbf{M}=$ migratorio y $\mathbf{R}=$ residente). 